\title{
Thermodynamic modelling of phase equilibria in Al-Ga-P-As system
}

\author{
S ACHARYA and J P HAJRA* \\ Department of Metallurgy, Indian Institute of Science, Bangalore 560 012, India \\ MS received 20 September 2004
}

\begin{abstract}
A generalized thermodynamic expression of the liquid Al-Ga-P-As alloys is used in conjunction with the solid solution model in determining the solid-liquid equilibria at $1173 \mathrm{~K}$ and $1273 \mathrm{~K}$. The liquid solution model contains thirtyseven parameters. Twentyfour of them pertain to those of the six constituent binaries, twelve refer to the specific ternary interactions. Additionally the liquid solution model also contains a specific quaternary interaction parameter. The latter has been evaluated here based on the experimental data available in the literature. The present research shows an excellent agreement between the derived and experimental values at $1173 \mathrm{~K}$ and $1273 \mathrm{~K}$ for the system. The article also presents a comparison between the evaluated values with those based on the regular solution model for the liquid alloys.
\end{abstract}

Keywords. Excess free energy; liquid solution model; Al-Ga-P-As system; solid-liquid equilibria; solid alloys; quaternary alloy.

\section{Introduction}

The compounds and the solid solutions of group III-V elements are extensively used in optoelectronic and high speed electronic devices fabrication. Since the need of the electronic industries is very specific in terms of composition, reliable description of phase equilibria is considered to be extremely relevant for the purpose. The evaluation of the phase equilibria of the system is based on appropriate thermodynamic functions for the liquid alloys and the solid solutions involved in the system. It has been reported by earlier investigators that the thermodynamic behaviour of solid solution may adequately be interpreted through an extended form of regular solution model (Pollack et al 1975). However, the corresponding liquidus compositions of higher order systems are poorly represented using a regular solution model (Pollack et al 1975). In the present investigation, therefore, a multiparameter function is adopted for interpretation of thermodynamic behaviour of the liquid alloys in the system.

According to Lupis (1983), a higher order system is considered to be composed of summation of the interactions of the constituent binaries along with the specific ternary and quaternary interactions. In the present research, the integral excess free energy function of the binary alloys are analysed using four-parameter equation. Six constituent binaries in the system provide twentyfour such constants ( $a_{1}$ to $a_{24}$ ). Similarly, three specific ternary interactions pertaining to four ternaries constitute twelve constants $\left(b_{1}\right.$ to $b_{12}$ ) in the system. Additionally, it has been shown subsequently that appropriate quaternary interaction has also been included in the function for more reliable definition of the equilibrium properties of the system. For a binary system, the excess free energy function is represented

*Author for correspondence (jph@metalrg.iisc.ernet.in) isothermally (Hajra and Mazumdar 1991) as

$$
\frac{\Delta G^{x s}}{R T}=x_{1} x_{2}\left\{a_{1} x_{1}+a_{2} x_{2}+x_{1} x_{2}\left(a_{3} x_{1}+a_{4} x_{2}\right)\right\} \text {. }
$$

The integral excess free energy for a binary system may also be expressed by Redlich-Kister polynomial (Redlich and Kister 1948) as

$$
\Delta G^{x s}=x_{1} x_{2}\left\{L_{0}+L_{1}\left(x_{1}-x_{2}\right)+L_{2}\left(x_{1}-x_{2}\right)^{2}+L_{3}\left(x_{1}-x_{2}\right)^{3}\right\} .
$$

The interrelationship between the four-parameter and Redlich-Kister polynomial may be expressed as

$$
\begin{aligned}
& R T^{*} a_{1}=L_{0}+L_{1}+L_{2}+L_{3}, \\
& R T^{*} a_{2}=L_{0}-L_{1}+L_{2}-L_{3}, \\
& R T^{*} a_{3}=-4\left(L_{2}+L_{3}\right), \\
& R T^{*} a_{4}=4\left(L_{3}-L_{2}\right) .
\end{aligned}
$$

Since the literature data pertaining to the constituent binaries of the system are expressed in terms of RedlichKister polynomial, the latter values have been transformed to their corresponding ' $a$ ' parameters to be used in the present investigation through (1).

According to the convention as mentioned above, the present formulation for the quaternary system consists of the $1-2,1-3,1-4,2-3,2-4,3-4$ binaries and four ternaries, viz. 1-2-3, 1-2-4, 1-3-4 and 2-3-4. The components of the quaternary system, Al-Ga-P-As have been designated as 1, 2, 3 and 4 , respectively. The integral excess free energy function based on this concept may be represented as

$$
\begin{aligned}
& \frac{\Delta G^{x s}}{R T}=x_{1} x_{2}\left\{a_{1} x_{1}+a_{2} x_{2}+x_{1} x_{2}\left(a_{3} x_{1}+a_{4} x_{2}\right)\right\}+ \\
& x_{1} x_{3}\left\{a_{5} x_{1}+a_{6} x_{3}+x_{1} x_{3}\left(a_{7} x_{1}+a_{8} x_{3}\right)\right\}+ \\
& x_{1} x_{4}\left\{a_{9} x_{1}+a_{10} x_{4}+x_{1} x_{4}\left(a_{11} x_{1}+a_{12} x_{4}\right)\right\}+ \\
& x_{2} x_{3}\left\{a_{13} x_{2}+a_{14} x_{3}+x_{2} x_{3}\left(a_{15} x_{2}+a_{16} x_{3}\right)\right\}+
\end{aligned}
$$




$$
\begin{aligned}
& x_{2} x_{4}\left\{a_{17} x_{2}+a_{18} x_{4}+x_{2} x_{4}\left(a_{19} x_{2}+a_{20} x_{4}\right)\right\}+ \\
& x_{3} x_{4}\left\{a_{21} x_{3}+a_{22} x_{4}+x_{3} x_{4}\left(a_{23} x_{3}+a_{24} x_{4}\right)\right\}+ \\
& x_{1} x_{2} x_{3}\left\{b_{1} x_{1}+b_{2} x_{2}+b_{3} x_{3}\right\}+x_{1} x_{2} x_{4}\left\{b_{4} x_{1}+b_{5} x_{2}+b_{6} x_{4}\right\} \\
& +x_{1} x_{3} x_{4}\left\{b_{7} x_{1}+b_{8} x_{3}+b_{9} x_{4}\right\}+ \\
& x_{2} x_{3} x_{4}\left\{b_{10} x_{2}+b_{11} x_{3}+b_{12} x_{4}\right\}+x_{1} x_{2} x_{3} x_{4}\{c\}
\end{aligned}
$$

where the mole fractions of the components $\mathrm{Al}, \mathrm{Ga}, \mathrm{P}$ and As are designated as $x_{1}, x_{2}, x_{3}$ and $x_{4}$, respectively and $c$ denotes the specific quaternary interaction in the system.

The partial excess properties of each of the components of the quaternary system are deduced based on the relations between the partials and the integral excess free energy function as expressed by the following relations

$$
\begin{aligned}
& R T \ln \gamma_{1}=\Delta G^{x s}-x_{2} \frac{\partial \Delta G^{x s}}{\partial x_{2}}-x_{3} \frac{\partial \Delta G^{x s}}{\partial x_{3}}-x_{4} \frac{\partial \Delta G^{x s}}{\partial x_{4}}, \\
& R T \ln \gamma_{2}=\Delta G^{x s}+\left(1-x_{2}\right) \frac{\partial \Delta G^{x s}}{\partial x_{2}}-x_{3} \frac{\partial \Delta G^{x s}}{\partial x_{3}}-x_{4} \frac{\partial \Delta G^{x s}}{\partial x_{4}},
\end{aligned}
$$

$R T \ln \gamma_{3}=\Delta G^{x s}-x_{2} \frac{\partial \Delta G^{x s}}{\partial x_{2}}+\left(1-x_{3}\right) \frac{\partial \Delta G^{x s}}{\partial x_{3}}-x_{4} \frac{\partial \Delta G^{x s}}{\partial x_{4}}$,

$R T \ln \gamma_{4}=\Delta G^{x s}-x_{2} \frac{\partial \Delta G^{x s}}{\partial x_{2}}-x_{3} \frac{\partial \Delta G^{x s}}{\partial x_{3}}+\left(1-x_{4}\right) \frac{\partial \Delta G^{x s}}{\partial x_{4}}$

Differentiating (3) with respect to $x_{2}, x_{3}, x_{4}$ respectively, we get the following three sets of relations

$$
\begin{aligned}
& \frac{1}{R T}\left(\frac{\partial \Delta G^{x s}}{\partial x_{2}}\right)=a_{1}\left\{\left(1-3 x_{2}-x_{3}-x_{4}\right)\left(1-x_{2}-x_{3}-x_{4}\right)\right\}+ \\
& a_{2}\left\{x_{2}\left(2-3 x_{2}-2 x_{3}-2 x_{4}\right)\right\}+a_{3}\left\{x_{2}\left(2-5 x_{2}-2 x_{3}-2 x_{4}\right)\right. \\
& \left.\left(1-x_{2}-x_{3}-x_{4}\right)^{2}\right\}+a_{4}\left\{x_{2}^{2}\left(3-5 x_{2}-3 x_{3}-3 x_{4}\right)\right. \\
& \left.\left(1-x_{2}-x_{3}-x_{4}\right)\right\}-a_{5}\left\{2 x_{3}\left(1-x_{2}-x_{3}-x_{4}\right)\right\}-a_{6} x_{3}^{2}-a_{7} \\
& \left\{3 x_{3}^{2}\left(1-x_{2}-x_{3}-x_{4}\right)^{2}\right\}-a_{8}\left\{2 x_{3}^{3}\left(1-x_{2}-x_{3}-x_{4}\right)\right\}-a_{9} \\
& \left\{2 x_{4}\left(1-x_{2}-x_{3}-x_{4}\right)\right\}-a_{10} x_{4}^{2}-a_{11}\left\{3 x_{4}^{2}\left(1-x_{2}-x_{3}-x_{4}\right)^{2}\right\}- \\
& a_{12}\left\{2 x_{4}^{3}\left(1-x_{2}-x_{3}-x_{4}\right)\right\}+a_{13}\left(2 x_{2} x_{3}\right)+a_{14} x_{3}^{2}+a_{15} \\
& \left(3 x_{2}^{2} x_{3}^{2}\right)+a_{16}\left(2 x_{2} x_{3}^{3}\right)+a_{17}\left(2 x_{2} x_{4}\right)+a_{18} x_{4}^{2}+a_{19}\left(3 x_{2}^{2} x_{4}^{2}\right)+a_{20} \\
& \left(2 x_{2} x_{4}^{3}\right)+b_{1}\left\{x_{3}\left(1-3 x_{2}-x_{3}-x_{4}\right)\left(1-x_{2}-x_{3}-x_{4}\right)\right\}+ \\
& b_{2}\left\{x_{2} x_{3}\left(2-3 x_{2}-2 x_{3}-2 x_{4}\right)\right\}+b_{3}\left\{x_{3}^{2}\left(1-2 x_{2}-x_{3}-x_{4}\right)\right\} \\
& +b_{4}\left(2 x_{2} x_{3} x_{4}\right)+b_{5}\left(x_{3}^{2} x_{4}\right)+b_{6}\left(x_{3} x_{4}^{2}\right)-b_{7}\left(x_{3}^{2} x_{4}\right)-b_{8}\left(x_{3} x_{4}^{2}\right)- \\
& b_{9}\left\{2 x_{3} x_{4}\left(1-x_{2}-x_{3}-x_{4}\right)\right\}+b_{10}\left\{x_{4}^{2}\left(1-2 x_{2}-x_{3}-x_{4}\right)\right\}+ \\
& b_{11}\left\{x_{4}\left(1-3 x_{2}-x_{3}-x_{4}\right)\left(1-x_{2}-x_{3}-x_{4}\right)\right\}+ \\
& b_{12}\left\{x_{2} x_{4}\left(2-3 x_{2}-2 x_{3}-2 x_{4}\right)\right\}+c\left\{\left(1-x_{2}-x_{3}-x_{4}\right)\right. \\
& \left.x_{3} x_{4}-x_{2} x_{3} x_{4}\right\},
\end{aligned}
$$

$\frac{1}{R T}\left(\frac{\partial \Delta G^{x s}}{\partial x_{3}}\right)=-a_{1}\left\{2 x_{2}\left(1-x_{2}-x_{3}-x_{4}\right)\right\}-a_{2}\left(2 x_{2}^{2}\right)-$

$a_{3}\left\{3 x_{2}^{2}\left(1-x_{2}-x_{3}-x_{4}\right)^{2}\right\}-a_{4}\left\{2 x_{2}^{3}\left(1-x_{2}-x_{3}-x_{4}\right)\right\}+$

$a_{5}\left\{\left(1-x_{2}-3 x_{3}-x_{4}\right)\left(1-x_{2}-x_{3}-x_{4}\right)\right\}+a_{6}$

$\left\{x_{3}\left(2-2 x_{2}-3 x_{3}-2 x_{4}\right)\right\}+a_{7}\left\{x_{3}\left(2-2 x_{2}-5 x_{3}-2 x_{4}\right)\right.$

$\left.\left(1-x_{2}-x_{3}-x_{4}\right)^{2}\right\}+a_{8}\left\{x_{3}^{2}\left(3-3 x_{2}-5 x_{3}-3 x_{4}\right)\right.$

$\left.\left(1-x_{2}-x_{3}-x_{4}\right)\right\}-a_{9}\left\{2 x_{4}\left(1-x_{2}-x_{3}-x_{4}\right)\right\}-a_{10} x_{4}^{2}-a_{11}$

$\left\{3 x_{4}^{2}\left(1-x_{2}-x_{3}-x_{4}\right)^{2}\right\}-a_{12}\left\{2 x_{4}^{3}\left(1-x_{2}-x_{3}-x_{4}\right)\right\}+$

$a_{13}\left(x_{2}^{2}\right)+a_{14}\left(2 x_{2} x_{3}\right)+a_{15}\left(2 x_{2}^{3} x_{3}\right)+a_{16}\left(3 x_{2}^{2} x_{3}^{2}\right)+a_{21}\left(2 x_{3} x_{4}\right)+$

$a_{22} x_{4}^{2}+a_{23}\left(3 x_{3}^{2} x_{4}^{2}\right)+a_{24}\left(2 x_{3} x_{4}^{3}\right)+b_{1}\left\{x_{2}\left(1-x_{2}-3 x_{3}-x_{4}\right)\right.$

$\left.\left(1-x_{2}-x_{3}-x_{4}\right)\right\}+b_{2}\left\{x_{2}^{2}\left(1-x_{2}-2 x_{3}-2 x_{4}\right)\right\}+$

$b_{3}\left\{x_{2} x_{3}\left(2-2 x_{2}-3 x_{3}-2 x_{4}\right)\right\}+b_{4}\left(x_{2}^{2} x_{4}\right)+b_{5}\left(2 x_{2} x_{3} x_{4}\right)+$

$b_{6}\left(x_{2} x_{4}^{2}\right)+b_{7}\left\{x_{3} x_{4}\left(2-2 x_{2}-3 x_{3}-2 x_{4}\right)\right\}+$

$b_{8}\left\{x_{4}^{2}\left(1-x_{2}-2 x_{3}-x_{4}\right)\right\}+b_{9}\left\{x_{4}\left(1-x_{2}-3 x_{3}-x_{4}\right)\right.$

$\left.\left(1-x_{2}-x_{3}-x_{4}\right)\right\}-b_{10}\left(x_{2} x_{4}^{2}\right)-b_{11}\left\{2 x_{2} x_{4}\left(1-x_{2}-x_{3}-x_{4}\right)\right\}-$

$b_{12}\left(x_{2}^{2} x_{4}\right)+c\left\{\left(1-x_{2}-x_{3}-x_{4}\right) x_{2} x_{4}-x_{2} x_{3} x_{4}\right\}$,

$\frac{1}{R T}\left(\frac{\partial \Delta G^{x s}}{\partial x_{4}}\right)=-a_{1}\left\{2 x_{2}\left(1-x_{2}-x_{3}-x_{4}\right)\right\}-a_{2}\left(x_{2}^{2}\right)-$

$a_{3}\left\{3 x_{2}^{2}\left(1-x_{2}-x_{3}-x_{4}\right)^{2}\right\}-a_{4}\left\{2 x_{2}^{3}\left(1-x_{2}-x_{3}-x_{4}\right)\right\}-$

$a_{5}\left\{2 x_{3}\left(1-x_{2}-x_{3}-x_{4}\right)\right\}-a_{6}\left(x_{3}^{2}\right)-a_{7}\left\{3 x_{3}^{2}\left(1-x_{2}-x_{3}-x_{4}\right)^{2}\right\}-$

$a_{8}\left\{2 x_{3}^{3}\left(1-x_{2}-x_{3}-x_{4}\right)\right\}+a_{9}\left\{\left(1-x_{2}-x_{3}-3 x_{4}\right)\left(1-x_{2}-x_{3}-x_{4}\right)\right\}+$ $a_{10}\left\{x_{4}\left(2-2 x_{2}-2 x_{3}-3 x_{4}\right)\right\}+a_{11}\left\{x_{4}\left(2-2 x_{2}-2 x_{3}-5 x_{4}\right)\right.$

$\left.\left(1-x_{2}-x_{3}-x_{4}\right)\right\}+a_{12}\left\{x_{4}^{2}\left(3-3 x_{2}-3 x_{3}-5 x_{4}\right)\right.$

$\left.\left(1-x_{2}-x_{3}-x_{4}\right)\right\}+a_{17}\left(x_{2}^{2}\right)+a_{18}\left(2 x_{2} x_{4}\right)+a_{19}\left(2 x_{2}^{3} x_{4}\right)+$

$a_{20}\left(3 x_{2}^{2} x_{4}^{2}\right)+a_{21}\left(x_{3}^{2}\right)+a_{22}\left(2 x_{3} x_{4}\right)+a_{23}\left(2 x_{3}^{3} x_{4}\right)+a_{24}\left(3 x_{3}^{2} x_{4}^{2}\right)-$

$b_{1}\left\{2 x_{2} x_{3}\left(1-x_{2}-x_{3}-x_{4}\right)\right\}-b_{2}\left(x_{2}^{2} x_{3}\right)-b_{3}\left(x_{2} x_{3}^{2}\right)+$

$b_{4}\left(x_{2}^{2} x_{3}\right)+b_{5}\left(x_{2} x_{3}^{2}\right)+b_{6}\left(2 x_{2} x_{3} x_{4}\right)+b_{7}\left\{x_{3}^{2}\left(1-x_{2}-x_{3}-2 x_{4}\right)\right\}+$

$b_{8}\left\{x_{3} x_{4}\left(2-2 x_{2}-2 x_{3}-3 x_{4}\right)\right\}+b_{9}\left\{x_{3}\left(1-x_{2}-x_{3}-3 x_{4}\right)\right.$

$\left.\left(1-x_{2}-x_{3}-x_{4}\right)\right\}+b_{10}\left\{x_{2} x_{4}\left(2-2 x_{2}-2 x_{3}-3 x_{4}\right)\right\}+b_{11}$

$\left\{x_{2}\left(1-x_{2}-x_{3}-3 x_{4}\right)\left(1-x_{2}-x_{3}-x_{4}\right)\right\}+$

$b_{12}\left\{x_{2}^{2}\left(1-x_{2}-x_{3}-2 x_{4}\right)\right\}+c\left\{\left(1-x_{2}-x_{3}-x_{4}\right) x_{3} x_{4}-x_{2} x_{3} x_{4}\right\}$,

substituting (8), (9) and (10) in (4), (5), (6) and (7), the partials of the components of the Al-Ga-P-As system may be obtained.

\section{Discussion}

In the present investigation, the phase equilibria is evaluated by using the present generalized model, (3) for the liquid phase and a nine-parameter model developed by Onda and Ito (1987) for the solid alloys. At equilibrium, 
the chemical potential of a component in solid phase equals to that of the component of the liquid phase. The latter is expressed as

$$
\mu_{i}^{l}=\mu_{i}^{s},
$$

where, $i=1$ to 4 .

The chemical potential of the components are related to the corresponding activities as

$$
\mu_{i}^{o s}+R T \ln a_{i}^{s}=\mu_{i}^{o l}+R T \ln a_{i}^{l} .
$$

The solidus composition in the system is governed by the solid solution of the compounds AlP, AlAs, GaP and GaAs. The activities of the compounds in equilibrium with the corresponding solid components of the system may be expressed as

$$
\left(\mu_{i j}^{(s)}-\mu_{i}^{(s)}-\mu_{j}^{(s)}\right)=0,
$$

where $i j$ is the compound with $i$ and $j$ as the constituent elements. These may be expanded into the following expression

$$
\begin{aligned}
& \left(\mu_{i j}^{0(s)}-\mu_{i}^{0(s)}-\mu_{j}^{0(s)}\right)+R T \ln a_{i j}^{(s)}-R T \ln a_{i}^{(s)}- \\
& R T \ln a_{j}^{(s)}=0 .
\end{aligned}
$$

By combining the above equations with (12), we obtain

$$
\begin{aligned}
& \left(\mu_{i j}^{0(s)}-\mu_{i}^{0(s)}-\mu_{j}^{0(s)}\right)+R T \ln a_{i j}^{(s)}=R T \ln a_{i}^{(l)}+ \\
& R T \ln a_{j}^{(l)}-\left(\mu_{i}^{0(s)}-\mu_{i}^{0(l)}\right)-\left(\mu_{j}^{0(s)}-\mu_{j}^{0(l)}\right),
\end{aligned}
$$

where $\mu^{0(s)}$ and $\mu^{0(l)}$ of the pure components and pure compounds are obtained from the literature compiled by Ansara et al (1994). $x_{i}^{s}$ and $x_{i}^{l}$ are the corresponding mole fractions pertaining to the solid and liquid alloys in the system.

For the system, under present investigation viz. $\mathrm{Al}_{x}$ $\mathrm{Ga}_{1-x} \mathrm{P}_{y} \mathrm{As}_{1-y}, \Delta G^{x s}$ may be written for the solid phase in accordance with Onda and Ito (1987) as

$$
\begin{aligned}
& \Delta G^{x s}=x y d_{1}+x(1-y) d_{2}+(1-x) y d_{3}+(1-x)(1-y) d_{4} \\
& +x(1-x) y^{2} d_{5}+x(1-x)(1-y)^{2} d_{6} \\
& +x^{2} y(1-y) d_{7}+(1-x)^{2} y(1-y) d_{8}+x(1-x) y(1-y) d_{9},
\end{aligned}
$$

where, $x$ and $y$ refer to the compositional parameters pertaining to the solid solutions as expressed by the formulation, $\mathrm{Al}_{x} \mathrm{Ga}_{1-x} \mathrm{P}_{y} \mathrm{As}_{1-y}$. The constants, $d_{1}$ through $d_{9}$ describe the thermodynamic behaviour of the solid phase and the choice of these parameters are based on the following pair wise interactions of the components of the system (AlAs, AlP, GaAs and GaP), $d_{1} \rightarrow \mathrm{AlP}, d_{2} \rightarrow \mathrm{AlAs}$, $d_{3} \rightarrow \mathrm{GaP}, \quad d_{4} \rightarrow \mathrm{GaAs}, \quad d_{5} \rightarrow \mathrm{AlP}-\mathrm{GaP}, \quad d_{6} \rightarrow \mathrm{AlAs}-$ GaAs, $d_{7} \rightarrow$ AlP-AlAs, $d_{8} \rightarrow$ GaP-GaAs, $d_{9} \rightarrow$ AlGaPAs.

The corresponding partials in the solid alloys are derived based on (5) through (7) and (14) using a similar procedure as has been adopted for the liquid alloys. The partials of the solid solution based on the above may be expressed as,

$$
\begin{aligned}
& R T \ln \gamma_{\mathrm{AlP}}=d_{1}(x+y-2 x y)+d_{2}(1-x-y+2 x y)+d_{3} \\
& (1-x-2 y+2 x y)+d_{4}(-1+x+2 y-2 x y)+d_{5} \\
& \left(2 x y+y^{2}-2 x^{2} y-6 x y^{2}+5 x^{2} y^{2}\right)+d_{6} \\
& \left(2 x y+x^{2}-5 x^{2} y-2 x y^{2}+5 x^{2} y^{2}\right)+d_{7}(1-2 x+5 y+ \\
& \left.x^{2}+5 y^{2}+10 x y-5 x^{2} y-10 x y^{2}+5 x^{2} y^{2}\right)+ \\
& d_{8}\left(1-4 x+3 x^{2}-2 y+10 x y-8 x^{2} y-6 x y^{2}+y^{2}+5 x^{2} y^{2}\right)+ \\
& d_{9}\left(x+y-6 x y-x^{2}-y^{2}+5 x^{2} y+6 x y^{2}-5 x^{2} y^{2}\right),
\end{aligned}
$$

$$
\begin{aligned}
& R T \ln \gamma_{\mathrm{AlAs}}=d_{1}(x-y)+d_{2}(-1+x+y)+d_{3}(1-x)+ \\
& d_{4}(1-x)+d_{5}\left(2 x y-2 x^{2} y-y^{2}+x^{2} y^{2}\right)+ \\
& d_{6}\left(-2 x y+x^{2}+2 x^{2} y-x^{2} y+x^{2} y^{2}\right)+ \\
& d_{7}\left(1-2 x-y+2 x y+x^{2}+y^{2}-x^{2} y-2 x y^{2}+x^{2} y^{2}\right)+ \\
& d_{8}\left(-1-x^{2}+2 x+2 y-2 x y-y^{2}+x^{2} y^{2}\right)+ \\
& d_{9}\left(x-x^{2}-y+x^{2} y+y^{2}-x^{2} y^{2}\right)
\end{aligned}
$$

$$
\begin{aligned}
& R T \ln \gamma_{\mathrm{GaP}}=d_{1}(x+y)+d_{2}(1-x-y)+d_{3}(1-x)+ \\
& d_{4}(-1+x)+d_{5}\left(2 x y-2 x^{2} y+y^{2}-2 x y^{2}+x^{2} y^{2}\right)+ \\
& d_{6}\left(x^{2}+2 x y-3 x^{2} y-2 x y^{2}+x^{2} y^{2}\right)+ \\
& d_{7}\left(1-2 x+x^{2}-3 y+6 x y+y^{2}-3 x^{2} y-2 x y^{2}+x^{2} y^{2}\right)+ \\
& d_{8}\left(1-4 x-2 y+y^{2}+6 x y+3 x^{2}-4 x^{2} y-2 x y^{2}+x^{2} y^{2}\right)+ \\
& d_{9}\left(x-x^{2}+y-4 x y-y^{2}+3 x^{2} y+2 x y^{2}-x^{2} y^{2}\right),
\end{aligned}
$$

$$
\begin{aligned}
& R T \ln \gamma_{\mathrm{GaAs}}=d_{1}(-x+y)+d_{2}(1+x-y)+d_{3}(-1+x)+ \\
& d_{4}(1-x)+d_{5}\left(-2 x y+y^{2}+2 x^{2} y-2 x y^{2}+x^{2} y^{2}\right)+ \\
& d_{6}\left(2 x y-x^{2}+x^{2} y-2 x y^{2}+x^{2} y^{2}\right)+ \\
& d_{7}\left(-1+2 x+y-2 x y-x^{2}+y^{2}+x^{2} y-2 x y^{2}+x^{2} y^{2}\right)+ \\
& d_{8}\left(1-2 y+2 x y-x^{2}+y^{2}-2 x y^{2}+x^{2} y^{2}\right)+ \\
& d_{9}\left(-x+y+x^{2}-y^{2}-x^{2} y+2 x y^{2}-x^{2} y^{2}\right) .
\end{aligned}
$$

Each of the components in the liquid phase and those of the corresponding solid alloys are substituted in (13) for the description of the solid-liquid equilibria in the system. A computer programme was developed to obtain the regressional values by least squares technique using the experimental data (Llegems and Panish 1974) in the system. The binary and ternary parameters involved in (3) are used from the available literature data (Ansara et al 1994; Ishida et al 1989). Although the values of the parameters for the solid alloys, viz. $d_{5}$ to $d_{8}$, are available at 1173 and 
$1273 \mathrm{~K}$ in the literature (Ishida et al 1989), $d_{1}, d_{2}, d_{3}, d_{4}$ and $d_{9}$ along with the specific interaction parameter $c$ have been evaluated by the above mentioned method. Incorporating these values, the phase equilibria has been calculated at $1173 \mathrm{~K}$ and $1273 \mathrm{~K}$.

Table 1 compiles the binary and ternary interaction parameters that are available from the literature at $1173 \mathrm{~K}$ and $1273 \mathrm{~K}$ (Ansara et al 1994; Ishida et al 1989). The regressional values of the solid interaction parameters for
(14) along with the specific quaternary interaction parameter at $1173 \mathrm{~K}$ and $1273 \mathrm{~K}$ are listed in table 2 . For the purpose of comparison tables 3-5 list the experimental (Llegems and Panish 1974) and their corresponding calculated compositional values for the solid and liquid phases at equilibrium. The derived values of the present investigation are in closer agreement with the experimental data relative to those of the regular solution model. The use of multiparameter function of the liquid alloys is, therefore,

Table 1. Interaction parameters from Ansara et al (1994) and Ishida et al (1989) at $1173 \mathrm{~K}$ and $1273 \mathrm{~K}$.

\begin{tabular}{|c|c|c|c|c|c|}
\hline \multirow{2}{*}{$\begin{array}{l}\text { Binary } \\
\text { parameter }\end{array}$} & \multicolumn{2}{|c|}{$\begin{array}{l}\text { Parameter } \\
\quad(R T)\end{array}$} & \multirow{2}{*}{$\begin{array}{c}\text { Ternary } \\
\text { parameter }\end{array}$} & \multicolumn{2}{|c|}{$\begin{array}{l}\text { Parameter } \\
\quad(R T)\end{array}$} \\
\hline & $1173 \mathrm{~K}$ & $1273 \mathrm{~K}$ & & $1173 \mathrm{~K}$ & $1273 \mathrm{~K}$ \\
\hline$a_{1}$ & $0 \cdot 00631$ & -0.02289 & $\mathrm{~b}_{1}$ & 0 & 0 \\
\hline$a_{2}$ & $-0 \cdot 11338$ & $-0 \cdot 13143$ & $\mathrm{~b}_{2}$ & 0 & 0 \\
\hline$a_{3}$ & $-0 \cdot 13105$ & $-0 \cdot 12075$ & $\mathrm{~b}_{3}$ & 0 & 0 \\
\hline$a_{4}$ & $-0 \cdot 13105$ & $-0 \cdot 12075$ & $b_{4}$ & $-6 \cdot 79982$ & $5 \cdot 49088$ \\
\hline$a_{5}$ & -13.69735 & $-12 \cdot 01003$ & $b_{5}$ & $-6 \cdot 79982$ & $5 \cdot 49088$ \\
\hline$a_{6}$ & $-13 \cdot 69735$ & $-12 \cdot 01003$ & $b_{6}$ & $-6 \cdot 79982$ & 5.49088 \\
\hline$a_{7}$ & 0 & 0 & $\mathrm{~b}_{7}$ & 0 & 0 \\
\hline$a_{8}$ & 0 & 0 & $\mathrm{~b}_{8}$ & 0 & 0 \\
\hline$a_{9}$ & $-5 \cdot 71825$ & $-5 \cdot 59184$ & $\mathrm{~b}_{9}$ & 0 & 0 \\
\hline$a_{10}$ & $-5 \cdot 71825$ & $-5 \cdot 59184$ & $b_{10}$ & -0.52295 & -0.48187 \\
\hline$a_{11}$ & 0 & 0 & $b_{11}$ & -0.52295 & -0.48187 \\
\hline$a_{12}$ & 0 & 0 & $b_{12}$ & -0.52295 & -0.48187 \\
\hline$a_{13}$ & $-1 \cdot 01125$ & -0.931808 & & & \\
\hline$a_{14}$ & $-1 \cdot 01125$ & -0.931808 & & & \\
\hline$a_{15}$ & 0 & 0 & & & \\
\hline$a_{16}$ & 0 & 0 & & & \\
\hline$a_{17}$ & $-3 \cdot 66425$ & $-3 \cdot 41714$ & & & \\
\hline$a_{18}$ & $-2 \cdot 60303$ & $-2 \cdot 44026$ & & & \\
\hline$a_{19}$ & 0 & 0 & & & \\
\hline$a_{20}$ & 0 & 0 & & & \\
\hline$a_{21}$ & $0 \cdot 59424$ & 0.54756 & & & \\
\hline$a_{22}$ & $0 \cdot 22904$ & $0 \cdot 21108$ & & & \\
\hline$a_{23}$ & 0 & 0 & & & \\
\hline$a_{24}$ & 0 & 0 & & & \\
\hline
\end{tabular}

Table 2. Regression values.

\begin{tabular}{lccc}
\hline & & \multicolumn{2}{c}{ Parameter $(R T)$} \\
\cline { 3 - 4 } Phase & Parameter & $1173 \mathrm{~K}$ & $1273 \mathrm{~K}$ \\
\hline \multirow{2}{*}{ Solid } & $d_{1}$ & $1.0937 \times 10^{2}$ & 0.5171 \\
& $d_{2}$ & $-4.2713 \times 10^{1}$ & $15 \cdot 0521$ \\
& $d_{3}$ & $3.5956 \times 10^{17}$ & $7.2455 \times 10^{16}$ \\
& $d_{4}$ & $3.5956 \times 10^{17}$ & $7.2455 \times 10^{16}$ \\
& $d_{5}$ & $0^{*}$ & $0^{*}$ \\
& $d_{6}$ & $0.417542^{*}$ & $0.384742^{*}$ \\
& $d_{7}$ & $0^{*}$ & $0^{*}$ \\
& $d_{8}$ & $0^{*}$ & $0^{*}$ \\
Liquid & $d_{9}$ & $1.1767 \times 10^{1}$ & -25.0920 \\
\hline & $c$ & $3.0614 \times 10^{4}$ & $-1.5121 \times 10^{5}$ \\
\hline
\end{tabular}

*Indicates the availability of data in literature (Ishida et al 1989). 
Table 3. Comparison of the solidus compositional data with the calculated values at $1173 \mathrm{~K}$ and $1273 \mathrm{~K}$.

\begin{tabular}{|c|c|c|c|c|c|c|c|c|c|c|}
\hline Temp. (K) & $x_{1}^{1}$ & $x_{2}^{1}$ & $x_{3}^{1}$ & $x_{4}^{1}$ & $x_{\text {expt }}^{s}$ & $\begin{array}{c}x_{\text {cal }}^{s} \\
\text { Present } \\
\text { study }\end{array}$ & $\begin{array}{c}x_{\mathrm{cal}}^{s} \\
\text { Llegems \& } \\
\text { Panish } \\
(1974)\end{array}$ & $y_{\text {expt }}^{s}$ & $\begin{array}{c}y_{\text {cal }}^{s} \\
\text { Present } \\
\text { study }\end{array}$ & $\begin{array}{c}y_{\text {cal }}^{s} \\
\text { Llegems \& } \\
\text { Panish } \\
(1974)\end{array}$ \\
\hline \multirow[t]{10}{*}{1173} & 0.0010 & 0.9576 & 0.0015 & 0.0399 & $0 \cdot 1852$ & $0 \cdot 1789$ & $0 \cdot 2013$ & $0 \cdot 2977$ & $0 \cdot 2993$ & $0 \cdot 3143$ \\
\hline & $0 \cdot 0010$ & 0.9668 & 0.0023 & 0.0299 & $0 \cdot 1687$ & $0 \cdot 2038$ & 0.2290 & 0.5092 & 0.5024 & 0.5197 \\
\hline & $0 \cdot 0010$ & 0.9757 & 0.0033 & $0 \cdot 0200$ & $0 \cdot 1951$ & $0 \cdot 2321$ & 0.2715 & 0.6307 & 0.6342 & 0.6441 \\
\hline & 0.0010 & 0.9505 & 0.0039 & 0.0099 & $0 \cdot 2376$ & $0 \cdot 2799$ & $0 \cdot 3140$ & $0 \cdot 8167$ & $0 \cdot 8385$ & $0 \cdot 8448$ \\
\hline & 0.0010 & 0.9945 & 0.0045 & $0 \cdot 0001$ & $0 \cdot 3410$ & $0 \cdot 3352$ & $0 \cdot 3565$ & 0.9959 & 0.9813 & 0.9713 \\
\hline & $0 \cdot 0010$ & 0.9853 & 0.0039 & 0.0097 & $0 \cdot 2390$ & $0 \cdot 2774$ & $0 \cdot 3167$ & $0 \cdot 8194$ & 0.8289 & 0.8502 \\
\hline & 0.0025 & 0.9571 & 0.0005 & 0.0399 & $0 \cdot 3648$ & $0 \cdot 3415$ & $0 \cdot 3178$ & $0 \cdot 1588$ & $0 \cdot 1528$ & $0 \cdot 1484$ \\
\hline & 0.0025 & 0.9660 & 0.0015 & $0 \cdot 0300$ & $0 \cdot 3816$ & $0 \cdot 3632$ & $0 \cdot 3952$ & 0.4310 & 0.4143 & 0.4032 \\
\hline & 0.0025 & 0.9755 & 0.0020 & 0.0201 & 0.4592 & 0.4626 & 0.4785 & 0.6211 & 0.6282 & 0.6388 \\
\hline & 0.0025 & 0.9944 & 0.0030 & 0.0001 & 0.5613 & 0.5578 & 0.5777 & 0.9715 & 0.9728 & 0.9959 \\
\hline \multirow{6}{*}{1273} & $0 \cdot 0010$ & 0.9846 & 0.0143 & $0 \cdot 0001$ & $0 \cdot 2844$ & $0 \cdot 2731$ & 0.2567 & 0.9960 & 0.9956 & 0.9940 \\
\hline & 0.0010 & 0.9314 & 0.0078 & 0.0598 & $0 \cdot 1401$ & $0 \cdot 1535$ & $0 \cdot 1725$ & 0.5793 & 0.5614 & 0.5874 \\
\hline & 0.0025 & 0.9322 & 0.0050 & 0.0603 & $0 \cdot 2874$ & $0 \cdot 3084$ & $0 \cdot 3109$ & 0.4864 & 0.4993 & 0.5157 \\
\hline & 0.0025 & 0.9506 & 0.0070 & 0.0391 & $0 \cdot 3495$ & $0 \cdot 3386$ & 0.3585 & 0.6740 & $0 \cdot 6810$ & 0.7133 \\
\hline & 0.0025 & 0.9693 & $0 \cdot 0088$ & 0.0196 & $0 \cdot 3544$ & $0 \cdot 3652$ & 0.4008 & $0 \cdot 8496$ & 0.8373 & $0 \cdot 8684$ \\
\hline & 0.0025 & 0.9867 & 0.0106 & $0 \cdot 0002$ & 0.4285 & $0 \cdot 4101$ & 0.4539 & 0.9973 & 0.9917 & 0.9928 \\
\hline
\end{tabular}

Table 4. Comparison of the liquidus compositional data with the calculated values at $1173 \mathrm{~K}$.

\begin{tabular}{|c|c|c|c|c|c|c|c|c|c|c|c|c|c|}
\hline \multirow[b]{2}{*}{$x_{1}^{l} \operatorname{expt}$} & \multicolumn{2}{|c|}{$x_{1 \text { assume }}^{l}$} & \multirow[b]{2}{*}{$x_{2}^{l}$ expt } & \multicolumn{2}{|c|}{$x_{2}^{l}$ cal } & \multirow[b]{2}{*}{$x_{3}^{l}$ expt } & \multicolumn{2}{|c|}{$x_{3}^{l}$ cal } & \multicolumn{3}{|c|}{$x_{4 \text { cal }}^{l}$} & \multirow[b]{2}{*}{$x_{\text {expt }}^{s}$} & \multirow[b]{2}{*}{$y_{\text {expt }}^{s}$} \\
\hline & $\begin{array}{l}\text { Present } \\
\text { study }\end{array}$ & $\begin{array}{c}\text { Llegems } \\
\text { \& Panish } \\
\text { (1974) }\end{array}$ & & $\begin{array}{l}\text { Present } \\
\text { study }\end{array}$ & $\begin{array}{l}\text { Llegems } \\
\text { \& Panish } \\
\text { (1974) }\end{array}$ & & $\begin{array}{l}\text { Present } \\
\text { study }\end{array}$ & $\begin{array}{l}\text { Llegems } \\
\text { \& Panish } \\
\text { (1974) }\end{array}$ & $x_{4}^{l}$ expt & $\begin{array}{l}\text { Present } \\
\text { study }\end{array}$ & $\begin{array}{l}\text { Llegems } \\
\text { \& Panish } \\
\text { (1974) }\end{array}$ & & \\
\hline 010 & & & 16 & & & 5 & & & & & & 52 & 977 \\
\hline & $0 \cdot 0010$ & $0 \cdot 0010$ & 0 & 660 & 0.9710 & & 25 & 0.0 & & 0.0289 & & & 92 \\
\hline $0 \cdot 0010$ & $0 \cdot 0010$ & $0 \cdot 0010$ & 0.9757 & 698 & 0.9813 & & 0.0032 & 0.0030 & & 0.0198 & & 51 & 0.6307 \\
\hline $0 \cdot 0010$ & $0 \cdot 0010$ & $0 \cdot 0010$ & $0 \cdot 9505$ & 615 & 0.9855 & 0.0039 & $0 \cdot 0038$ & 0.0037 & $0 \cdot 0099$ & $0 \cdot 0098$ & 0.0097 & $0 \cdot 2376$ & $0 \cdot 8167$ \\
\hline 0.0010 & 0.0010 & $0 \cdot 0010$ & 0.9945 & 0.9941 & 0.9844 & $0 \cdot 0045$ & 0.0044 & 0.0044 & $0 \cdot 0001$ & $0 \cdot 0002$ & 0.0001 & $0 \cdot 3410$ & 0.9959 \\
\hline 0.0010 & $0 \cdot 0010$ & $0 \cdot 0010$ & 0.9853 & 0.9875 & 0.9849 & 0.0039 & 0.0038 & 0.0037 & $0 \cdot 0097$ & $0 \cdot 0096$ & 0.0094 & $0 \cdot 2390$ & $0 \cdot 8194$ \\
\hline 0.0025 & 0.0025 & 0.0025 & & 0.9580 & 0.9565 & 0.0005 & $0 \cdot 0006$ & 0.0005 & 0.0399 & 0.0400 & $0 \cdot 0404$ & $0 \cdot 3648$ & $0 \cdot 1588$ \\
\hline 0.0025 & $0 \cdot 0025$ & 0.0025 & & 0.9690 & 0.9664 & & $0 \cdot 0014$ & 0.0013 & 0.0300 & 0.0298 & & $0 \cdot 3816$ & 0.4310 \\
\hline 0.0025 & 0.0025 & 0.0025 & & & & & $0 \cdot 0021$ & & & 0.0206 & & & 0.6211 \\
\hline 0.0025 & $0 \cdot 0025$ & $0 \cdot 0025$ & 0.9944 & 0.9969 & 0.9927 & 0.0030 & 0.0031 & 0.0028 & $0 \cdot 0001$ & $0 \cdot 0001$ & 0.0002 & 0.5613 & 0.9715 \\
\hline
\end{tabular}

Table 5. Comparison of the liquidus compositional data with the calculated values at $1273 \mathrm{~K}$.

\begin{tabular}{|c|c|c|c|c|c|c|c|c|c|c|c|c|c|}
\hline \multirow[b]{2}{*}{$x_{1 \text { expt }}^{l}$} & \multicolumn{2}{|c|}{$x_{1 \text { assume }}^{l}$} & \multirow[b]{2}{*}{$x_{2}^{l}$ expt } & \multicolumn{2}{|c|}{$x_{2 \text { calc }}^{l}$} & \multirow[b]{2}{*}{$x_{3}^{l}$ expt } & \multicolumn{2}{|c|}{$x_{3 \text { calc }}^{l}$} & \multirow[b]{2}{*}{$x_{4 \text { expt }}^{l}$} & \multicolumn{2}{|c|}{$x_{4 \text { calc }}^{l}$} & \multirow[b]{2}{*}{$x_{\text {expt }}^{s}$} & \multirow[b]{2}{*}{$y_{\text {expt }}^{s}$} \\
\hline & $\begin{array}{l}\text { Present } \\
\text { study }\end{array}$ & $\begin{array}{l}\text { Llegems } \\
\text { \& Panish } \\
\text { (1974) }\end{array}$ & & $\begin{array}{l}\text { Present } \\
\text { study }\end{array}$ & $\begin{array}{l}\text { Llegems } \\
\text { \& Panish } \\
\text { (1974) }\end{array}$ & & $\begin{array}{c}\text { Present } \\
\text { study }\end{array}$ & $\begin{array}{c}\text { Llegems } \\
\text { \& Panish } \\
\text { (1974) }\end{array}$ & & $\begin{array}{l}\text { Present } \\
\text { study }\end{array}$ & $\begin{array}{l}\text { Llegems } \\
\text { \& Panish } \\
\text { (1974) }\end{array}$ & & \\
\hline 0.0010 & 10 & 0 & 846 & $T$ & & 143 & & & 0 & & & & 960 \\
\hline 0.0010 & 0.0010 & 0.0010 & 14 & 0.9416 & 0.9591 & 0078 & 0.0075 & 0.0072 & $0 \cdot 0$ & 0.5917 & 91 & & 0.5793 \\
\hline 0.0025 & 0.0025 & 0.0025 & 0.9322 & 0.9320 & 0.9319 & 0.0050 & $0 \cdot 0051$ & 0.0051 & $0 \cdot 0603$ & $0 \cdot 0601$ & $0 \cdot 0606$ & 71 & 0.5144 \\
\hline 0.0025 & 0.0025 & $0 \cdot 0025$ & 0.9506 & 0.9530 & $0 \cdot 9517$ & $0 \cdot 0070$ & $0 \cdot 0071$ & 0.0069 & $0 \cdot 0391$ & $0 \cdot 0401$ & 0.0399 & $0 \cdot 3630$ & 0.7101 \\
\hline 0.0025 & $0 \cdot 0025$ & 0.0025 & 0.9693 & 0.9659 & 0.9691 & 0.0088 & 0.0085 & 0.0087 & $0 \cdot 0196$ & $0 \cdot 0201$ & 0.0197 & 0.4096 & $0 \cdot 8684$ \\
\hline 0.0025 & $0 \cdot 0025$ & 0.0025 & 0.9867 & 0.9815 & 0.9889 & $0 \cdot 0106$ & $0 \cdot 0101$ & 0.0106 & $0 \cdot 0002$ & $0 \cdot 0003$ & $0 \cdot 0005$ & 0.4481 & 0.9863 \\
\hline
\end{tabular}




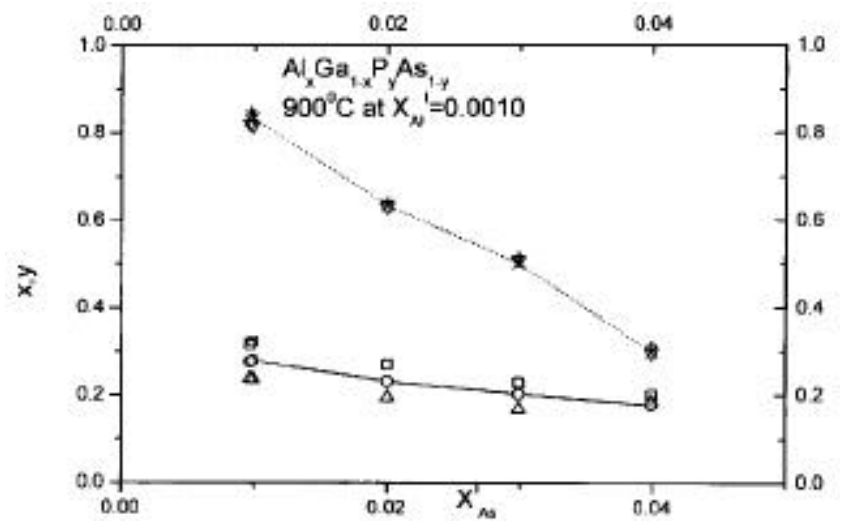

Figure 1. $900^{\circ} \mathrm{C}$ Solidus isotherms at constant, $X_{\mathrm{Al}}^{l}=0.0010$ (For $x$ : $\Delta$, experimental (Llegems and Panish 1974); - O -, present study; $\square$, Llegems and Panish's (1974) model; and for y: $\nabla$, experimental (Llegems and Panish 1974); ... ..., present study; +, Llegems and Panish's (1974) model).

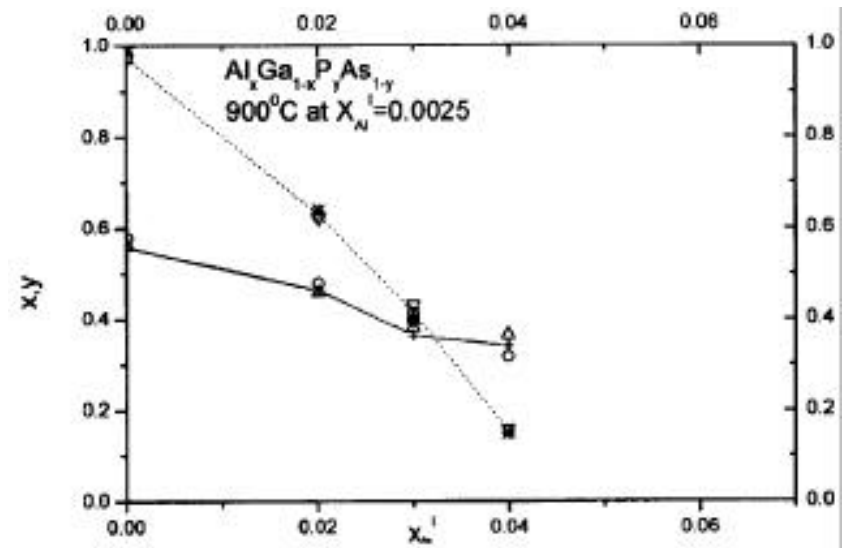

Figure 2. $900^{\circ} \mathrm{C}$ Solidus isotherms at constant, $X_{\mathrm{Al}}{ }^{l}=0.0025$ (For $x$ : $\Delta$, experimental (Llegems and Panish 1974); -+-, present study; O, Llegems and Panish's (1974) model; and for $y: \nabla$, experimental (Llegems and Panish 1974); ... $\square$..., present study; *, Llegems and Panish's (1974) model).

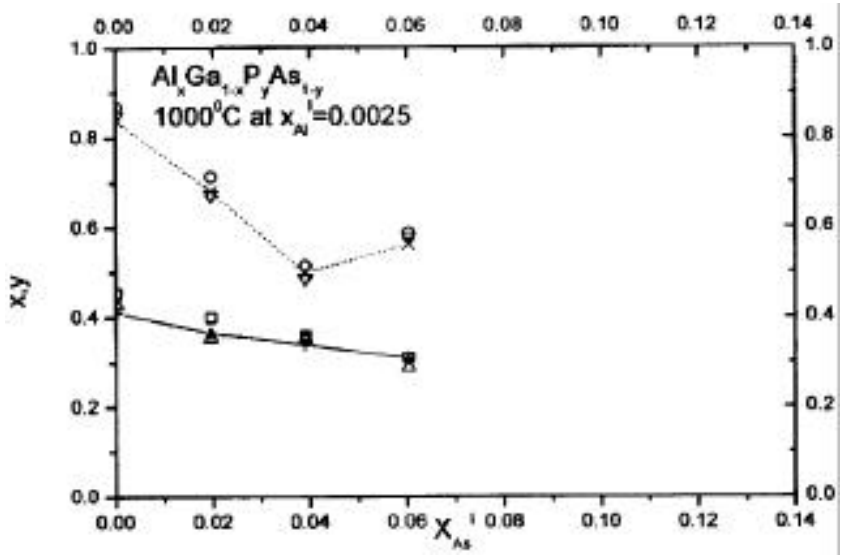

Figure 3. $1000^{\circ} \mathrm{C}$ Solidus isotherms at constant $X_{\mathrm{Al}}^{l}=0.0025$, (For $x$ : $\Delta$, experimental (Llegems and Panish 1974); -+-, present study; $\square$, Llegems and Panish's (1974) model; and for $y: \nabla$, experimental (Llegems and Panish (1974); ... ..., present study; O, Llegems and Panish's (1974) model). considered to be adequate in establishing the phase equilibria in the system. Figures 1-6 exhibit the calculated values of solidus and liquidus composition based on the present research and those obtained through the simple solution model for the purpose of comparison. The figures show that the derived values of solidus composition based on the two models are in agreement with the experimental data. However, the derived values of the liquidus composition based on the present research are much closer to the experimental data for $\mathrm{Al}$ rich alloys at lower temperature than those derived based on the simple solution model. Since specific compositional analysis is necessary in the electronic industries in order to develop a particular electronic grade of materials for certain special applications, more exact determination of the liquidus compositions seems appropriate for the purpose. For dilute solutions, the effect of the higher order interaction bet-

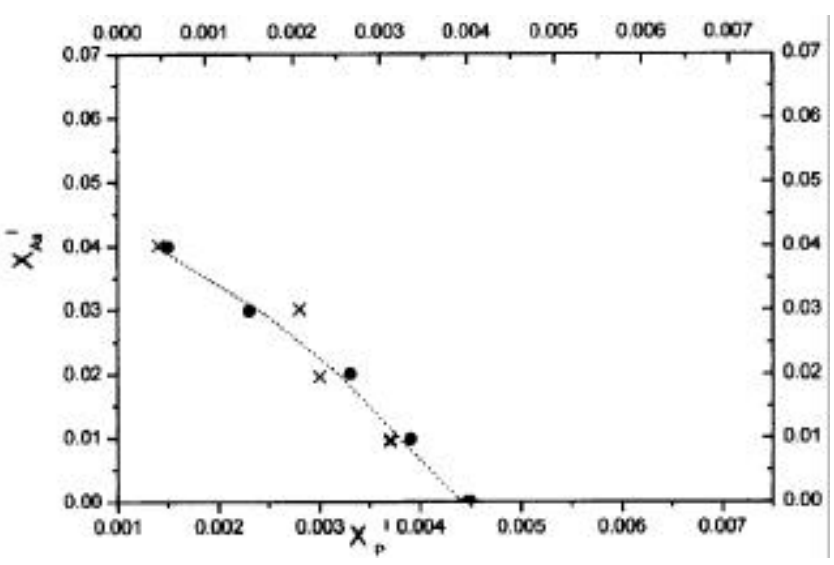

Figure 4. $900^{\circ} \mathrm{C}$ Liquidus isotherms in the $\mathrm{Al}-\mathrm{Ga}-\mathrm{P}-\mathrm{As}$ system at $X_{\mathrm{Al}}^{l}=0.0010(\bullet$, experimental (Llegems and Panish 1974); ....., present study; $\times$, Llegems and Panish's (1974) model).

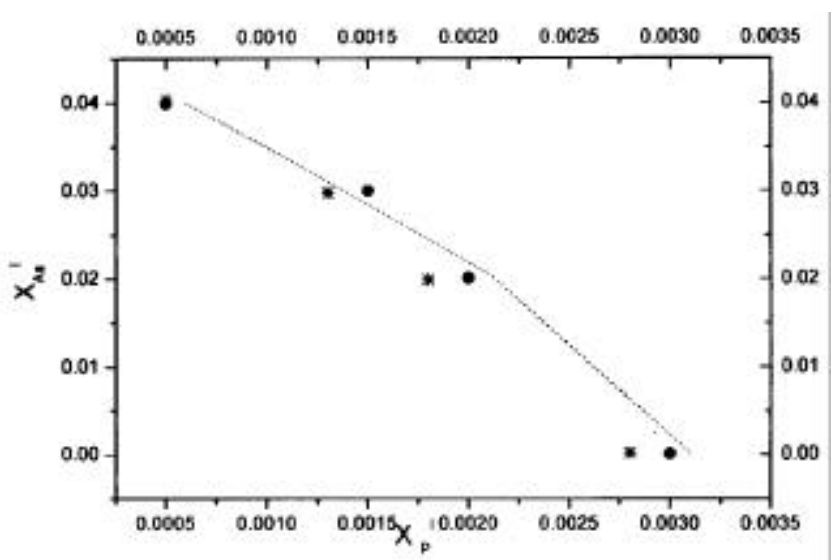

Figure 5. $900^{\circ} \mathrm{C}$ Liquidus isotherms in the $\mathrm{Al}-\mathrm{Ga}-\mathrm{P}-\mathrm{As}$ system at $X_{\mathrm{Al}}^{l}=0.0025(\bullet$, experimental (Llegems and Panish 1974); ....., present study; *, Llegems and Panish's (1974) model). 


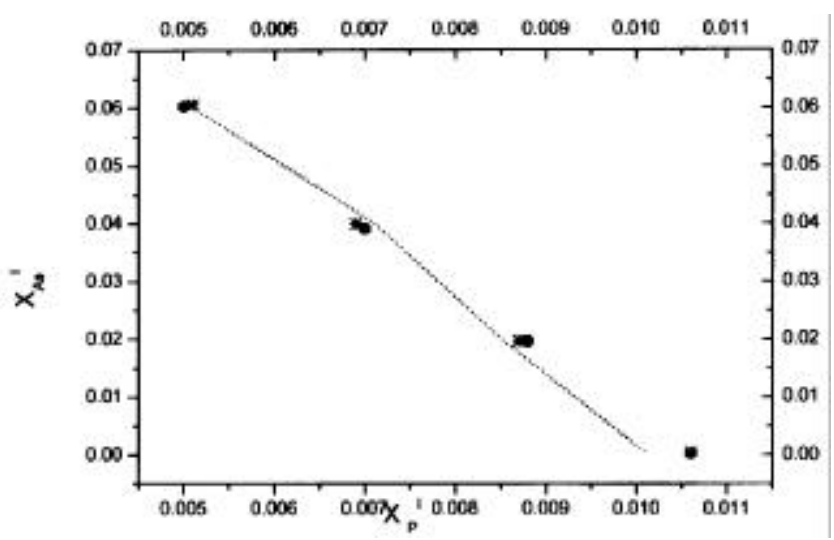

Figure 6. $1000^{\circ} \mathrm{C}$ Liquidus isotherms in the $\mathrm{Al}-\mathrm{Ga}-\mathrm{P}-\mathrm{As}$ system at $X_{\mathrm{Al}}^{l}=0.0025,(\bullet$, experimental (Llegems and Panish 1974); ....., present study; *, Llegems and Panish's (1974) model).

ween atoms are generally considered to be negligible. However, the role of higher order parameters are extremely necessary for appropriate description of equilibrium properties of the system without any compositional constraints.

\section{Conclusions}

The present research describes the isothermal section of the Al-Ga-P-As system at $1173 \mathrm{~K}$ and $1273 \mathrm{~K}$. The proposed liquid solution model is used along with a solid solution model by Onda and Ito (1987) to study the solidliquid equilibria in the Al-Ga-P-As system. The parameters for which the data are not available in the literature, have been determined through the regressional analysis of the solid-liquid equilibrium data of the system. Although the derived values of simple solution model for the solid solution is adequate in representing the solidus data, it departs somewhat from those of the liquidus compositions of the alloys. The use of multi parameter function in the present research complies with the specific requirement of more exact compositional description of the liquid alloys in the system (liquidus compositions).

\section{Acknowledgement}

The support and assistance to one of the authors (JPH) by the AICTE is gratefully acknowledged.

\section{References}

Ansara I et al 1994 Calphad 18177

Hajra J P and Mazumdar B 1991 Met. Trans. B22 583

Ishida K, Tokunga H, Ohtani H and Nishizawa T 1989 J. Crystal Growth 98140

Llegems M and Panish M B 1974 J. Phys. Chem. Solids 35409

Lupis C H P 1983 Chemical thermodynamics of materials (New York: Elsevier Science)

Onda T and Ito Ryoichi 1987 Jpn. J. Appl. Phys. 261241

Pollack M A, Nahory R E and Deas L V 1975 J. Electrochem. Soc. 1221550

Redlich O and Kister A 1948 Ind. Eng. Chem. 40345 